Review Article

\title{
Bedaquiline: a new weapon against MDR and XDR-TB
}

\author{
Harmanjit Singh $^{\mathrm{a} *}$, Navreet Kaur Natt ${ }^{\mathrm{b}}$, Nipunjot Garewal ${ }^{\mathrm{c}}$, Pugazhenthan $\mathbf{T}^{\mathrm{a}}$
}

${ }^{\mathrm{a}}$ Department of Pharmacology, PGIMER, Chandigarh, India, ${ }^{\mathrm{b}}$ Medical Officer (TB-control), District TB Hospital, Amritsar, Punjab, India, ${ }^{c}$ Department of Pharmacology, Punjab Institute of Medical Sciences, Jalandhar, Punjab, India

Received: 23 January 2013

Accepted: 27 January 2013

*Correspondence to:

Dr. Harmanjit Singh,

Email: harman_gmcp@yahoo.com

(C) 2013 Singh $\mathrm{H}$ et al. This is an openaccess article distributed under the terms of the Creative Commons Attribution License, which permits unrestricted use, distribution, and reproduction in any medium, provided the original work is properly cited.

\begin{abstract}
Multidrug-resistant tuberculosis (MDR-TB) is a global public health problem. It requires treatment with combination therapy consisting of four to six drugs including combinations of bactericidal and bacteriostatic drugs, usually for a period of 2 years. There is alarming rise in MDR and XDR-TB all over the world and better treatment options are needed to control the global MDR-TB and XDR-TB epidemic. Drugs which can shorten the treatment duration and which are free from serious adverse effects are urgently needed. Bedaquiline (TMC-207) is a newly FDA approved anti-TB drug, having unique mechanism of action i.e. causes inhibition of the proton pump activity of the ATP synthase in M. tuberculosis and targets the energy metabolism. It is found to active within macrophages, and is a promising agent in shortening the duration of anti- TB treatment. It is metabolized by CYP3A4, so interactions with inducers and inhibitors of this enzyme are expected. It has shown promising results in preclinical and clinical studies and it seems to be a good option for MDR and XDR-TB. Adverse effects reported in various studies were of mild nature except nausea which was the most commonly associated. Few cases of prolongation of QT intervals were reported, so it demands careful monitoring and use of bedaquiline as a reserve drug for patients in whom conventional regimens are not effective. Currently it is approved as part of combination therapy in adults of $\geq 18$ year with pulmonary MDR-TB. Long term studies are needed to explore its full safety profile.
\end{abstract}

Keywords: MDR, XDR, TB, Bedaquiline, TMC-207, Mycobacteria

\section{INTRODUCTION}

Multidrug-resistant tuberculosis (MDR-TB) has emerged as a global public health problem. It is resistant to at least isoniazid and rifampicin, the two most effective bactericidal drugs currently available for treatment of TB. ${ }^{1}$ It requires treatment with combination therapy consisting of four to six drugs including a fluoroquinolone and an injectable anti-TB agent, as well as bacteriostatic agents are administered for a prolonged period, usually up to 2 years. ${ }^{2}$ Moreover, this treatment is generally more toxic and more costly than the standardized treatment regimen used to treat drug susceptible TB. ${ }^{3,4}$

In recent surveys by the $\mathrm{WHO}$, it has been found that about $3.7 \%$ of new TB patients in the world have MDRTB strains. Levels are much higher in those previously treated - about $20 \%$. The frequency of MDR-TB varies substantially between countries. WHO estimated that
220,000-400,000 MDR-TB cases were notified in the world in 2011 and approximately $60 \%$ of these cases were found in Brazil, China, India, the Russian Federation and South Africa alone ("BRICS" countries). About $9 \%$ of MDR-TB cases were also found to have extensively drug-resistant TB (XDR-TB). As of October 2012, 84 countries had reported at least one XDR-TB case. $^{5}$

XDR-TB, a more severe form of MDR-TB which is also resistant to a fluoroquinolone and an injectable anti-TB agent, has emerged at an alarming rate and resulted in poor outcomes with the existing treatment. ${ }^{6}$ In a recent meta-analysis of XDR-TB patients, the treatment success was demonstrated only in $44 \%$ of patients, but mortality was in the range of $14 \%$ to $27 \%$. $^{7}$ In the presence of Human Immunodeficiency Virus (HIV) co-infection, mortality can exceed $70 \%$ among XDR-TB patients despite appropriate treatment for $\mathrm{HIV}^{8}$ Hence, better 
treatment options are needed to control the global MDRTB and XDR-TB epidemic.

Anti-TB therapy comprises combination of bactericidal and bacteriostatic drugs that prevent the development of resistance and improve the therapeutic outcome. It is very crucial to contain and treat the MDR-TB epidemic with new, shorter, and safe treatment regimens, which are easily accessible to the community. Such regimens should contain new drugs able to combat MDR- TB, and must be free from serious adverse effects and drug-drug interactions. $^{9,10}$ Our success will depend on the development of new anti-TB agents designed to achieve the following important objectives:

- Shorten treatment duration.

- Increase compliance by promoting intermittent therapy.

- Introduce new agents with novel mechanisms of action and activity against drug resistant TB.

- Decrease incidence of latent TB infection by developing safer, shorter duration treatment regimens.

\section{WHAT IS BEDAQUILINE?}

It is a new anti-TB agent (formerly known as TMC-207 or R207910), a diarylquinoline drug indicated as part of combination therapy in adults $(\geq 18$ years) with pulmonary MDR-TB, having a novel mechanism of action against mycobacterium tuberculosis. It is found to be effective against both drug susceptible and drug resistance $\mathrm{TB}$, including strains that are resistant to streptomycin, pyrazinamide, ethambutol and moxifloxacin. It has bactericidal and sterilizing activity against $M$. tuberculosis and other mycobacterial species, but little activity against other bacteria. The remarkable sterilizing capacity of this drug also makes it an attractive drug in the strategy of TB elimination. It is found to active within macrophages, and is a promising agent in shortening the duration of anti- TB treatment. ${ }^{11-13}$

\section{MECHANISM OF ACTION OF BEDAQUILINE ${ }^{12,14,15}$}

It has unique and specific antimycobacterial activity. It acts by targeting subunit $\mathrm{c}$ of the ATP synthase of $M$. tuberculosis, leading to inhibition of the proton pump activity of the ATP synthase. Thus, the compound targets bacillary energy metabolism. ATP synthase is a critical enzyme in the synthesis of ATP for M. tuberculosis. Binding of bedaquiline to the oligomeric and proteolipic subunit $\mathrm{c}$ of mycobacterial ATP synthase leads to inhibition of ATP synthesis. This leads to the death of mycobacteria. In contrast to several other bacteria, which can produce sufficient ATP by substrate-level phosphorylation and tolerate deletion of ATP synthase, ATP synthase has been proven to be essential for optimal growth in M. tuberculosis. Mutations in subunit $\mathrm{c}$ conferring resistance to TMC207 suggest a binding site in the central region of subunit $\mathrm{c}$.

\section{ABSORPTION, DISTRIBUTION, AND EXCRETION $^{12,15,16}$}

After oral dose of $400 \mathrm{mg}$ of, the $t_{\max }$ was found to be 4 hours, the $\mathrm{C}_{\max }$ was $5.5 \mathrm{mg} / \mathrm{L}$ after $400 \mathrm{mg} / \mathrm{day}$, and the $\mathrm{AUC}_{0-24}$ was $65 \mathrm{mg} \mathrm{h} / \mathrm{L}$. Based on these data, the clearance was around $6.2 \mathrm{~L} / \mathrm{h}$. A very interesting pharmacokinetic effect in humans is its long terminal half-life of 173 hours. This factor makes it suitable for intermittent drug administration. The AUC and the $\mathrm{C}_{\max }$ have shown a linear pharmacokinetic profile in both single-and multiple-dose studies, and the half-life is largely independent of the dose. CYP3A4 is the major CYP isoenzyme involved in the metabolism of bedaquiline and the formation of the $\mathrm{N}$-monodesmethyl metabolite (M2), which is 4 to 6-times less active in terms of antimycobacterial potency. The plasma protein binding of bedaquiline is $>99.9 \%$. The volume of distribution in the central compartment is estimated to be approximately $164 \mathrm{~L}$. Data from preclinical studies show that bedaquiline is mainly eliminated in feces. The urinary excretion of unchanged bedaquiline was $<0.001 \%$ of the dose in clinical studies, indicating that renal clearance of unchanged drug is insignificant. When the drug is taken with food exposure increases approximately two fold.

\section{PHARMACODYNAMICS AND ANTIMICROBIAL PROPERTIES ${ }^{12,14,15,17,18,19}$}

In vitro studies show that bedaquiline potently inhibits drug-sensitive, as well as drug-resistant mycobacterial $\mathrm{TB}$ at a minimal inhibitory concentration (MIC) of 0.03 $0.12 \mathrm{mg} / \mathrm{L}$. It has been suggested that dormant mycobacteria have lower ATP stores (these mycobacteria have mild ATP synthase activity which is necessary for their survival), so these bacteria becomes more vulnerable to further ATP depletion by bedaquiline even at nanomolar concentrations. This action makes it an effective sterilizing agent against mycobacteria and a superior member than other $1^{\text {st }}$ line anti-TB drugs. The drug has shown a considerable strong inhibitory effect against many of non-tubercular mycobacteria (NTM), e.g. M. avium and M. intracellulare, M. abscessus and $M$. ulcerans. In these conditions, it is almost as active as in mycobacteria tuberculosis. It also possesses good activity against MAC, M. leprae, $M$. bovis, M. marinum, $M$. kansasii, M. fortuitum, and M. szulgai. It has been found that it takes 2-4 days for its antibacterial activity to occur. This lag period may be due to its mechanism of action i.e. delayed ATP depletion and disruption of intracellular $\mathrm{pH}$ homeostasis. A significant bactericidal activity has been reported at days 4-7 after starting the treatment. 


\section{BACTERIAL RESISTANCE ${ }^{12,15,17,20}$}

Resistance has been found to be associated with two point mutations: D32V and A63P. This region of the gene encodes the membrane-spanning domain of the ATP synthase $c$ subunit. It has been found that the gene encoding for the subunit $\mathrm{c}$ of ATP synthase is atpE, and it has a highly conserved amino acid sequence. The presence of mutations (at position 63 where proline replaces alanine or at position 66 where methionine replaces leucine) of the $a t p E$ gene inhibits the binding of the drug to the $\mathrm{c}$ subunit of the ATP synthase enzyme. The drug is thought to have a binding pocket around amino acid residue Glu61. Mutations at these sites interfere with the access of bedaquiline to its target site residue 61 . This leads to either natural or acquired resistance. Natural resistance is mainly seen in NTM, while acquired resistance is mainly seen in $M$. tuberculosis.

\section{PRE-CLINICAL AND CLINICAL RESEARCH}

1. Andries et $\mathrm{al}^{14}$ demonstrated that bedaquiline potently inhibits both drug-sensitive and drugresistant Mycobacterium tuberculosis in vitro. In mice, the drug has shown superior bactericidal activities than isoniazid and rifampin by at least $1 \mathrm{log}$ unit. It also accelerated the time to complete culture conversion in some combinations. After single dose, mycobacterial growth was inhibited for at least 1 week. Plasma levels associated with efficacy in mice were well tolerated in healthy human volunteers.

2. Ibrahim et $\mathrm{al}^{21}$ evaluated the sterilizing activity of bedaquiline-containing combinations in the murine model of TB and they concluded that four months of treatment with bedaquiline containing regimens was as effective as the six month treatment with standard regimen and more effective than four months of treatment with moxifloxacin containing regimens. They concluded that supplementation of standard regimen (RHZ) with bedaquiline or substitution of bedaquiline for rifampin may shorten the treatment duration needed to cure TB in patients.

3. In a preclinical study done by Lenaerts et $\mathrm{al}^{22}$, it was concluded that 6 week treatment of guinea pigs with bedaquiline was highly effective and lead to complete eradication of the bacteria throughout both the primary and the secondary lesions in lung granulomas.

4. Lounis et $\mathrm{al}^{23}$ conducted a study in mice where they found that all bedaquiline containing regimens were significantly more active than the non- bedaquiline containing regimens after 1 month of therapy. When given for 2 months, bedaquiline alone was more active than the WHO standard first-line regimen and when it was combined with second-line drugs, the combinations were more active than the currently recommended regimen of MDR-TB. The culture negativity of both the lungs and spleen was reached after 2 months of treatment in almost all animals.

5. In a study on mice model, Zhang et $\mathrm{al}^{24}$ compared the efficacy of TMC207 alone and in combination with rifapentine with currently recommended control regimens as well as once-weekly rifapentine + isoniazid and daily rifapentine \pm isoniazid. They concluded that TMC207 exhibited sterilizing activity at least as strong as that of rifampin alone and similar to that of rifampin + isoniazid, but daily rifapentine +/- isoniazid was superior to TMC207. Addition of TMC207 to rifapentine did not improve the sterilizing activity of rifapentine in this model.

6. Veziris et $\mathrm{al}^{25}$ conducted a study where the mice were inoculated with $M$. tuberculosis and then treated with second-line drug combinations with or without the bedaquiline, and then followed without treatment for 3 more months to determine relapse rates. After addition of bedaquiline to this MDR TB regimen, the treatment duration needed to reach the same relapse rate dropped to 6 months. A similar relapse rate was also obtained with a 6-month completely oral regimen including bedaquiline, moxifloxacin and pyrazinamide but excluding both amikacin and ethionamide. They concluded that inclusion of bedaquiline in the WHO MDR-TB treatment regimen has the potential to further shorten the treatment duration and at the same time to simplify treatment by eliminating the need to include an injectable aminoglycoside.

7. In an early bactericidal study done by Rustomjee et $\mathrm{al}^{26}, 75$ treatment-naïve patients with smearpositive pulmonary TB were randomized to oncedaily oral bedaquiline ( $25 \mathrm{mg}, 100 \mathrm{mg}$, or $400 \mathrm{mg}$ ), $600 \mathrm{mg}$ rifampin, or $300 \mathrm{mg}$ isoniazid for 7 days. The bactericidal activity was expressed as the log (10) decrease in $\mathrm{CFU} / \mathrm{ml}$ sputum/day. Significant bactericidal activity of $\quad 400$ mg bedaquiline was observed from day 4 onward and was similar in magnitude to those of isoniazid and rifampin over the same period. Bedaquiline demonstrated bactericidal activity with a delayed onset and was very well tolerated, and no drugrelated serious adverse events occurred during the study.

8. In first stage of a phase 2, randomized, controlled trial done by Diacon et $\mathrm{al}^{27}, 47$ MDR-TB patients were randomly assigned to receive either bedaquiline in dose of $400 \mathrm{mg}$ daily for 2 weeks, followed by $200 \mathrm{mg}$ three times a week for 6 weeks (23 patients) or placebo (24 patients) in combination with a standard five-drug, second-line anti- TB regimen. The primary efficacy end point was the conversion of sputum cultures, in liquid broth, from positive to negative. After addition of bedaquiline to standard 
therapy, the time to conversion to a negative sputum culture was significantly reduced as compared with placebo and proportion of patients with conversion of sputum culture was significantly increased. No severe adverse effects were detected, and only nausea occurred significantly more frequently among patients in the bedaquiline group than among patients in the placebo group.

9. In second stage of the previous phase 2 trial, Diacon et $\mathrm{al}^{28}$ presented the 2-year follow-up results for a RCT of 47 MDR-TB patients treated with either the bedaquiline, or placebo. These treatments were added for the first 8 weeks of a background regimen. Bedaquiline lead to a significant reduction in the time to culture conversion over 24 weeks (hazard ratio, $2.253 ; 95 \% \mathrm{CI}, 1.08$ to $4.71 ; p=0.031$ ). Adverse effects occurred at almost similar frequencies in both groups except nausea which was significantly higher in bedaquiline group. Other adverse effects associated were: bilateral hearing impairment, extremity pain, acne, and noncardiac chest pain. During this trial only one patient receiving bedaquiline acquired resistance to companion drugs, but five patients receiving placebo ( $4.8 \%$ versus $21.7 \% ; p=0.18$ ) acquired resistance to companion drugs. In placebo group, 13 patients (54\%) discontinued the study prior to its completion and 10 patients receiving bedaquiline (44\%), discontinued the study before completion. They concluded that bedaquiline may be a very effective therapy for MDR-TB as it leads to more rapid sputum culture conversion and by prevents the development of resistance to the other drugs in the regimens.

10. In a study (Wallis et al) ${ }^{29}$, the early bactericidal activity of PNU-100480, TMC207, PA-824, SQ109, and pyrazinamide, was examined singly and in various combinations by using whole blood culture. These drugs were predicted to have cumulative activity comparable to standard TB therapy and further testing of these regimens was warranted.

\section{DOSAGE, ADVERSE DRUG REACTIONS, WARNINGS, DRUG INTERACTIONS AND CONTRAINDICATIONS}

\section{Dose}

Weeks 1-2: $400 \mathrm{mg}$ once daily with food, weeks 3-24: $200 \mathrm{mg} 3$ times per week with food, for a total dose of $600 \mathrm{mg}$ per week. ${ }^{30,31}$

The adverse events encountered with TMC207 are mild and include nausea in $26 \%$ of patients and diarrhea in $13 \%$ of patients, with others such as arthralgia, pain in extremities, and hyperuricemia in a small proportion of patients. $^{15}$
At a dose of $400 \mathrm{mg}$ daily, it was associated with a low rate of adverse events. The most frequent events reported were rash (7\%) with $100 \mathrm{mg}$ dose, and diarrhea (7\%) and somnolence $(7 \%)$ in the $400 \mathrm{mg}$ dose. Two patients treated with $400 \mathrm{mg}$, died 14 and 34 days after the end of bedaquiline treatment but neither death (one due to hemoptysis and the other from complications of TB and AIDS) was considered to be due to bedaquiline. The electrocardiogram showed increase in the QT interval, but no pathologically prolonged QT or corrected QT values were reported. Most of adverse events were of mild or moderate in nature; nausea, diarrhea, arthralgia, dizziness, hyperuricemia and eye disorders were more frequently observed in patients treated with bedaquiline. Incidence of nausea was significantly higher in bedaquiline group. ${ }^{12,27,28}$ However, only a limited number of patients have been exposed to this drug, so that the full side-effect profile is unclear. ${ }^{15}$

There are two important black box warnings for the use of bedaquiline i.e. prolongation of QT interval and an increased risk of death with bedaquiline as compared to placebo treatment. It should be only used when other treatment regimen are not effective or are not tolerated. ${ }^{32}$

Important precautions and contraindicated are and,27,28,30,31 $^{12,}$ ECG should be done before starting treatment and at least 2,12 , and 24 weeks after starting therapy.

Baseline serum potassium, calcium, and magnesium levels should be monitored and corrected if abnormal. There is Increased risk for QT prolongation if:

- The drug is coadministered with other QT prolonging drugs e.g. fluoroquinolones, macrolides etc.

- There is history of Torsade de Pointes or congenital long QT syndrome

- There are low levels of serum calcium, magnesium, or potassium

Discontinue bedaquiline if the following develops:

- Clinically significant ventricular arrhythmia

- $\quad$ QTcF interval $>500 \mathrm{~ms}$

Bedaquiline is metabolized by CYP3A4; concurrent use of strong CYP3A4 inducers (e.g., rifampin, rifapentine, rifabutin) should be avoided otherwise they may reduce the effect of bedaquiline and treatment failure can occur.

Coadministration with CYP3A4 inhibitors may increase systemic concentrations and result in increased adverse effects; concurrent use with strong CYP3A4 inhibitors e.g. ketoconazole and ciprofloxacin and other quinolones should be avoided, but if necessary it should not exceed more than 14 consecutive days. The drug has shown synergistic effects with pyrazinamide (in vitro). 
Use in pregnancy: It has been assigned pregnancy category B, no harm to the developing fetus was found in preclinical studies but studies in humans are lacking and additional data is required.

Hepatic Disease: usually, no dose adjustment is required in patients with mild or moderate hepatic impairment. It has not been studied in patients with severe hepatic disease and should be used with caution in these patients only when the benefits outweigh the risks.

Renal Disease: most of the studies were conducted in patients with normal renal functions, so adequate data is not available. No dose adjustment is required in patients with mild or moderate renal impairment, but should be used with caution in patients with severe renal disease.

There are no clinical data on the combined use of antiretroviral agents and bedaquiline in HIV/MDR-TB coinfected patients and only limited clinical data on the use of bedaquiline in HIV/MDR-TB coinfected patients not receiving antiretroviral therapy. ${ }^{30,31}$

Currently, it is recommended for use in patients of $\geq 18$ years of age, hence contraindicated in children. No adequate data in geriatric patients over the age 65 years is available, additional trials are required to establish its safety and efficacy in geriatric patients. ${ }^{30,31}$

\section{CONCLUSION}

In view of above discussion, it can be said that bedaquiline has shown definite beneficial effects in combination with other anti-TB drugs. Bedaquiline is the first new anti-TB drug to be approved by the FDA (on $28^{\text {th }}$ December, 2012) in more than 40 years. It was approved under the FDA's accelerated review program that allows promising drugs to be brought to market more quickly. ${ }^{32,33}$ It seems to be an attractive option for MDR and XDR- TB and shortening the duration of anti- TB therapy, but the drug has its own limitations and drawbacks e.g. adverse effects like prolongation of QT interval, nausea and drug interaction with CYP3A4 inducers and inhibitors. Therefore, careful use of this drug along with monitoring of the potential adverse effects and drug interactions becomes very important.

Funding: No funding sources

Competing interests: None declared

Ethical approval: Not required

\section{BEDAQUILINE: KEY FEATURES}

- First-in-class diarylquinoline active against drug-sensitive and drug-resistant TB.

- $\quad$ FDA approved $\left(28^{\text {th }}\right.$ Dec, 2012) for use in MDR-TB in patients $\geq 18$ years of age.

- Have novel mechanism of action i.e. inhibition of the proton pump activity of the ATP synthase in M. Tuberculosis.

- Promising agent in shortening the duration of the anti-TB treatment and hence reducing the global burden of the disease.

- Takes 4-7 days for onset of bactericidal activity

- Shown promising results in various preclinical and clinical studies.

- Nausea was found to be most significantly associated adverse effect. Diarrhea, arthralgia, dizziness, hyperuricemia and eye disorders were seen, but not significant

- Interacting potential with CYP3A4 inducer/inhibitor systems.

- Few cases of prolongation of QT interval and few deaths were reported (Black Box Warning) but neither was directly linked to the drug.

\section{REFERENCES}

1. Multidrug and extensively drug-resistant TB, WHO, 2010 Global Report. Available at http://whqlibdoc.who.int/publications/2010/9789241 599191_eng.pdf. Accessed 19 Jan 2013.

2. Falzon D, Jaramillo E, Schünemann HJ, Arentz M, Bauer M, Bayona J, et al. WHO guidelines for the programmatic management of drug-resistant tuberculosis: 2011 update. Eur Respir J 2011;38:516-28.
3. Diel R, Rutz S, Castell S, Schaberg T. Tuberculosis: cost of illness in Germany. Eur Respir J 2012;40:143-51.

4. Loddenkemper R, Sotgiu G, Mitnick CD. Cost of tuberculosis in the era of multidrug resistance: will it become unaffordable? Eur Respir J 2012;40:9-11.

5. MDR- TB, 2012 update. Available at http://www.who.int/tb/publications/

MDRFactSheet2012.pdf. Accessed 22 Jan 2013.

6. Sotgiu G, Ferrara G, Matteelli A, Richardson MD, Centis R, Toungoussova $\mathrm{O}$ et al. Epidemiology and clinical management of XDR-TB: a systematic review by TBNET. Eur Respir J 2009;33:871-81. 
7. Jacobson KR, Tierney DB, Jeon CY, Mitnick CD, Murray MB. Treatment outcomes among patients with extensively drug-resistant tuberculosis: systematic review and meta-analysis. Clin Infect Dis 2010;51:6-14.

8. Gandhi NR, Moll A, Sturm AW, Pawinski R, Govender T, Lalloo U, et al. Extensively drugresistant tuberculosis as a cause of death in patients coinfected with tuberculosis and HIV in a rural area of South Africa. Lancet 2006;368:1575-80.

9. Mitchison DA. Role of individual drugs in the chemotherapy of tuberculosis. Int $\mathrm{J}$ Tuberc Lung Dis 2000;4:796-806.

10. World Health Organization. 2011. Global tuberculosis control 2011. Available at: http://www.who.int/tb/publications/2011/en/index.ht ml. Accessed 19 Jan 2013.

11. Field SK, Fisher D, Jarand JM, Cowie RL. New treatment options for multidrug-resistant tuberculosis. Ther Adv Respir Dis 2012;6:255-268.

12. Matteelli A, Carvalho AC, Dooley KE, Kritski A. TMC207: the first compound of a new class of potent anti-tuberculosis drugs. Future Microbiol 2010;5:849-58.

13. Ahmad S. New Perspectives in the Diagnosis and Treatment of Tuberculosis. J Bacteriol Parasitol 2012;3:e114. doi:10.4172/2155-9597.1000e114.

14. Andries K, Verhasselt P, Guillemont J. E. G, Gohlmann HW. Neefs JM, Winkler H, et al. A diarylquinoline drug active on the ATP synthase of Mycobacterium tuberculosis. Science 2005;307:223-7.

15. Gumbo T. TMC-207 (R207910). In: Bruten L, Chabner BA, Knollmann BC, editors. Goodman and Gillman's The Pharmacological Basis of Therapeutics. 12th edn. New York, NY: McGraw Hill Companies; 2011:1561.

16. Van Heeswijk, R, Lachaert R, Leopold L, DeBeule $\mathrm{K}$, McNeeley D. The effect of CYP3A4 inhibition on the clinical pharmacokinetics of TMC207. Presented at: the 38th Union World Conference on Lung Health; Cape Town, South Africa. 8-12 November 2007; Poster PS-71358-71311.

17. Huitric E, Verhasselt P, Andries K, Hoffner SE. In vitro antimycobacterial spectrum of a diarylquinoline ATP-synthase inhibitor. Antimicrob Agents Chemother 2007;51:4202-4.

18. Shi L, Sohaskey CD, Kana BD, Dawes S, North RJ, Mizrahi V, et al. Changes in energy metabolism of Mycobacterium tuberculosis in mouse lung and in vitro conditions affecting aerobic respiration. Proc Natl Acad Sci U S A 2005;102:15629-34.

19. Koul A, Vranckx L, Dendouga N, Balemans W, Van den Wyngaert I, Vergauwen $\mathrm{K}$, et al. Diarylquinolines are bactericidal for dormant mycobacteria as a result of disturbed ATP homeostasis. J Biol Chem 2008;283:25273-80.

20. Petrella S, Cambau E, Chauffour A, Andries K, Jarlier V, Sougakoff W. Genetic basis for natural and acquired resistance of the diaryquinoline
R207910 in mycobacteria. Antimicrob Agents Chemother 2006;50:2853-6.

21. Ibrahim M, Truffot-Pernot C, Andries K, Jarlier V, Veziris N. Sterilizing activity of R207910 (TMC207) - containing regimens in the murine model of tuberculosis. Am J Respir Crit Care Med 2009;180:553-7.

22. Lenaerts AJ, Hoff D, Aly S, Ehlers S, Andries K, Cantarero L, et al. Location of persisting mycobacteria in a Guinea pig model of tuberculosis revealed by R207910. Antimicrob Agents Chemother 2007;51:3338-45.

23. Lounis N, Veziris N, Chauffour A, Truffot-Pernot C, Andries K, Jarlier V. Combinations of R207910 with drugs used to treat multidrug-resistant tuberculosis have the potential to shorten treatment duration. Antimicrob Agents Chemother 2006;50:3543-7.

24. Zhang T, Li SY, Williams KN, Andries K, Nuermberger EL. Short-course chemotherapy with TMC207 and rifapentine in a murine model of latent tuberculosis infection. Am J Respir Crit Care Med 2011;184:732-7.

25. Veziris N, Ibrahim M, Lounis N, Andries K, Jarlier $\mathrm{V}$. Sterilizing activity of second-line regimens containing TMC207 in a murine model of tuberculosis. PLoS One 2011;6:e17556.

26. Rustomjee R, Diacon AH, Allen J, Venter A, Reddy $\mathrm{C}$, Patientia RF et al. Early bactericidal activity and pharmacokinetics of the dairylquinoline TMC207 in treatment of pulmonary tuberculosis. Antimicrob Agents Chemother 2008;52:2831-5.

27. Diacon AH, Pym AP, Grobusch M, Patientia R, Rustomjee R, Page-Shipp L, et al. The diarylquinoline TMC207 for multidrug-resistant tuberculosis. N Engl J Med 2009;360:2397-405.

28. Diacon AH, Donald PR, Pym A, Grobusch M, Patientia RF, et al. Randomized pilot trial of eight weeks of bedaquiline (TMC207) treatment for multidrug-resistant tuberculosis: long-term outcome, tolerability, and effect on emergence of drug resistance. Antimicrob Agents Chemother 2012;56:3271-6.

29. Wallis RS, Jakubiec W, Mitton-Fry M, Ladutko L, Campbell S, Paige D et al. Rapid evaluation in whole blood culture of regimens for XDR-TB containing PNU-100480 (sutezolid), TMC207, PA824, SQ109, and pyrazinamide. PLoS One 2012;7:e30479.

30. Sirturo (bedaquiline fumarate). Available at http://dailymed.nlm.nih.gov/dailymed/ lookup.cfm?setid=1534c9ae-4948-4cf4-9f66222a99db6d0e.pdf. Accessed 20 Jan 2013.

31. Bedaquiline. Available
http://reference.medscape.com/drug/sirturobedaquiline-999799\#5. Accessed 20 Jan 2013.

32. FDA approves first drug to treat multi-drug resistant tuberculosis. Available at http://www.fda.gov/NewsEvents/Newsroom/PressA 
nnouncements/ucm333695.htm. Accessed 19 Jan 2013.

33. WHO to convene expert meeting on the use of bedaquiline for MDR-TB treatment. Available at http://www.tbonline.info/posts/2013/1/7/who- convene-expert-meeting-use-bedaquiline-mdr-tb-/. Accessed 21 Jan 2013.

doi:10.5455/2319-2003.ijbcp20130301

Cite this article as: Singh $\mathrm{H}$, Natt NK, Garewal N, Pugazhenthan T. Bedaquiline: a new weapon against MDR and XDR-TB. Int J Basic Clin Pharmacol 2013;2:96-102. 